\title{
Crystal Habit Modification Using Habit Modifiers
}

\author{
Satyawati S. Joshi \\ University of Pune, \\ India
}

\section{Introduction}

The synthesis of inorganic materials with a specific size and morphology has recently received much attention in the material science research area. Morphology control or morphogenesis is more important for the chemical industry than size control. Many routes have been reported to control the crystal growth and eventually modify the morphology of the crystals. For crystal-habit modification, crystals are grown in the presence of naturally occurring soluble additives, which usually adsorb or bind to the crystal faces and influence the crystal growth or morphology. A number of recent investigations show that such type of crystal-habit modifiers can be used to obtain inorganic crystals with organized assemblies. (Xu, et al. 2007, Yu \& Colfen 2004, \& Colfen, 2001).

The crystal-habit modifiers may be of a very diverse nature, such as multivalent cations, complexes, surface active agents, soluble polymers, biologically active macromolecules, fine particles of sparingly soluble salts, and so on. (Sarig et al.,1980) These crystal modifiers often adsorb selectively on to different crystal faces and retard their growth rates, thereby influencing the final morphology of the crystals. (Yu \& Colfen, 2004) The strategy that uses organic additives and/or templates with complex functionalization patterns to control the nucleation, growth, and alignment of inorganic crystals has been universally applied for the biomimetic synthesis of inorganic materials with complex forms. (Qi et al., 2000) The biomimetic process uses an organized supramolecular matrix and produces inorganic crystals with characteristic morphologies. (Xu et al., 2007\& Loste \&Meldrum, 2001) Understanding the mechanism involved in such a matrix-mediated synthesis has a great potential in the production of engineering materials. Thus, catalyst particles of controlled size and morphology, magnetic materials with appropriate anisotropy, highly porous materials, composites, and well-organized crystallite assemblies can be produced by this synthesis method. (Sinha etal.,2000) Using water-soluble polymers as crystal modifiers for controlled crystallization is widely expanding and becoming a benign route for controlling and designing the architectures of inorganic materials. (Yu \& Colfen, 2004) Investigators have used different double hydrophilic block copolymers, such as poly(ethylene glycol)block-poly(methacrylic acid), to control the morphology of a number of inorganic salts, namely, $\mathrm{CaCO}_{3}$, (Sedlak \& Colfen, 2001, Rudolff et al., 2002, Meng et al., 2007, Guo et al., 2006, He et al., 2006, Wang et al., 2005, Meldrum et al., 2007, Gorna et al.,2007, \& Colfen \&Qi, 2001) $\mathrm{BaCO}_{3}, \mathrm{CdCO}_{3}, \mathrm{MnCO}_{3}, \mathrm{PbCO}_{3}$, (Yu et al.,2003) $\mathrm{BaCrO}_{4}$, (Liu et al.2005\& Yu et al., 
2002) $\mathrm{BaSO}_{4}$, (Qi et al., 2000, Robinson et al.,2002, Wang et al., 2005,\& Yu et al., 2005) tolazamide, $\mathrm{Pb}-\mathrm{WO}_{4}$, (Kuldipkumar et al., 2005) and so forth. In the early stages, gel matrices have been used for the control of nucleation and morphology in aqueous solutionbased crystal growth. (Yu et al., 2007 Oaki \&Imai, 2003) Investigators have used poly(vinyl alcohol) (PVA)-, agar-, gelatin-, and pectin-based gel matrices to control the morphology of inorganic crystals such as $\mathrm{PbI}_{2}, \mathrm{AgI}, \mathrm{Ag}_{2} \mathrm{Cr}_{2} \mathrm{O}_{7}, \mathrm{PbSO}_{4}, \mathrm{PbCl}_{2}$, and so forth. (Henisch, 1988) The advantage of a gel medium is believed to be the reduction of the nucleation rate and suppression of convection. (Yu \& Colfen, 2004) The functional groups, such as amine, amide, carboxylic acid and so forth, are known to significantly influence the mineralization process. Among the reported common gel matrices used as crystal-habit modifiers, PVA is a water soluble synthetic polymer with excellent film-forming and emulsifying properties. PVA is a crystalline polymer with a monoclinic structure and is known for its biological activities. (Merrill \& Bassett, 1975) Also, PVA is reported to have been used for the morphology control of $\mathrm{K}_{2} \mathrm{Cr}_{2} \mathrm{O}_{7}, \mathrm{AgBr}$, and $\mathrm{CaCO}_{3}$, (Sinha, 2001) and even for the selective nucleation of $\mathrm{CaCO}_{3}$ polymorphs. (Lakshminarayanan, 2003) In this chapter, the results on morphological changes using polymers as habit modifiers are discussed on the basis of nucleation theory and growth process.

\section{Crystal habit}

Although crystals can be classified according to seven crystal systems, the relative sizes of the faces of a particular crystal can vary considerably. This variation is called a modification of habit.

\subsection{Crystal habit modifications}

\subsubsection{Crystal morphology and structure}

The morphology of a crystal depends on the growth rates of the different crystallographic faces. Some faces grow very fast and have little or no effect on the growth form; while slow growing faces have more influence. The growth of a given face is governed by the crystal structure and defects on one hand and by the environmental conditions on the other. (Mullin 2002)

A number of proposed mechanisms and theories have been put forth to predict the equilibrium form of a crystal. According to the Bravais rule, the important faces governing the crystal morphology are those with the highest reticular densities and greatest interplanar distances, $\mathrm{d}_{\mathrm{hkl}}$. Or in simpler terms, the slowest growing and most influential faces are the closest packed and have the lowest Miller indices. The surface theories suggest that the equilibrium form should be such that the crystal has a minimum total surface free energy per unit volume.

The crystals may grow rapidly, or be stunted, in one direction; thus an elongated growth of a prismatic habit gives a needle shaped crystal (acicular habit) and a stunted growth gives a flat plate-like crystal (tabular, platy or flaky habit). The relative growths of the faces of a crystal can be altered and often controlled by a number of factors. Rapid crystallization, produced by the sudden cooling or seeding of a supersaturated solution, may result in the formation of needle crystals. The growth of a crystal may be stunted in certain directions due to presence of impurities in the crystallizing solution. A change of solvent often changes the crystal habit. 


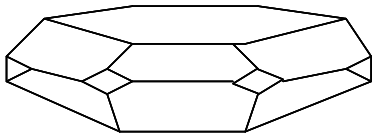

(a) Tabular

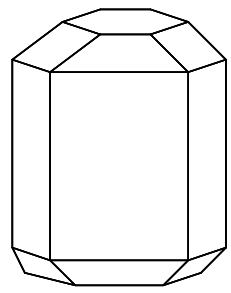

(b) Prismatic

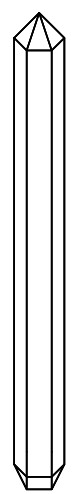

(c) Acicular

Fig. 1. Crystal habit illustrated on a hexagonal crystal

\subsubsection{Crystal surface structure}

The structure of a growing crystal face at its interface with the growth medium has been characterized by a quantity as surface roughness or surface entropy factor or the alpha factor defined by

$$
\mathrm{a}=\xi \Delta \mathrm{H} / \mathrm{kT}
$$

Where $\xi$ is an anisotropy factor related to the bonding energies in the crystal surface layers, $\Delta \mathrm{H}$ is the enthalpy of fusion and $\mathbf{k}$ is the Boltzmann constant. Values of a less than 2 are indicative of rough crystal surface which will allow continuous growth to proceed. The growth will be diffusion controlled and the face growth rates, v, will be linear with respect to the supersaturation, $\sigma$, i.e.

$$
v a \sigma
$$

For $a>5$, a smooth surface is indicated

\subsection{Effect of crystal size}

In order for crystallization to occur, there must exist in a solution a number of minute solid bodies, nuclei or seeds that act as a centre of crystallization, the classical theory of nucleation stemming from the work of Gibbs (1948) Volmer (1939) and others is based on the condensation of vapor to liquid and this treatment may be extended to crystallization from melts and solutions. Crystallization process can be explained on the basis of nucleation and growth process.

\subsubsection{Nucleation}

Schematically the nucleation steps are as shown below: 


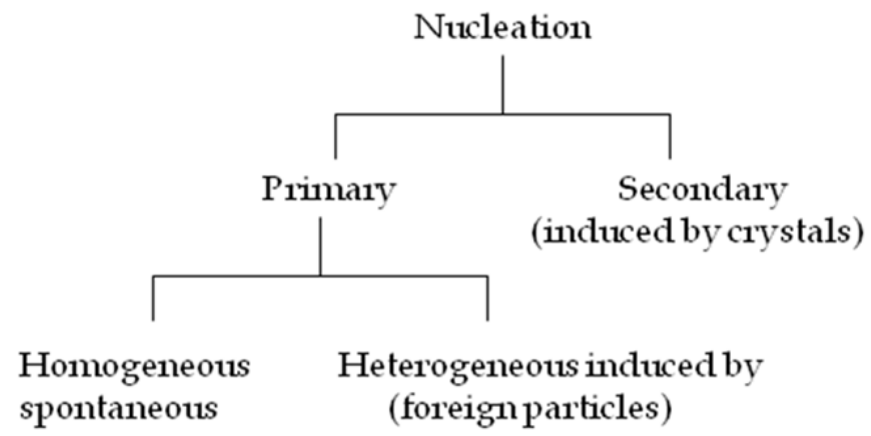

Let us consider the free energy changes associated with the process of homogenous nucleation. The overall excess free energy, $\Delta G$, between a small solid particles of solute ( assume here a sphere of radius $\mathrm{r}$ for simplicity) and the solute in solution is equal to the sum of surface excess free energy $\Delta G$ s i.e. excess free energy between the surface of the particles and the bulk of the particles, and the volume excess free energy, $\Delta \mathrm{Gv}$, i.e. the excess free energy between very large particles $(r=\infty)$ and the solute in the solution, $\Delta G$ s is a positive quantity, the magnitude of which is proportional to $\mathrm{r}^{2}$. In a supersaturated solution $\mathrm{Gv}$ is a negative quantity proportional to $\mathrm{r}^{3}$. Thus

$$
\begin{aligned}
& \Delta \mathrm{G}=\Delta \mathrm{G} s+\Delta \mathrm{G} v \\
& =4 \pi r^{2} \gamma+\frac{4}{3} \pi r^{3} \Delta \mathrm{G} v
\end{aligned}
$$

$\Delta \mathrm{Gv}$ also can be understood as free energy change of formation per unit volume $\gamma$ is the interfacial tension or surface energy.

$\Delta \mathrm{Gs}$ and $\Delta \mathrm{Gv}$ are opposite in sign and depend differently on $\mathrm{r}$. the free energy of formation, $\Delta \mathrm{G}$, passes through a maximum $\left(\Delta \mathrm{G}_{\text {crit }}\right)$ corresponds to critical nucleus, $\mathrm{r}_{\mathrm{c}}$. For a spherical cluster, it is obtained by setting $\mathrm{d} \Delta \mathrm{G} / \mathrm{dr}=0$

$$
\begin{gathered}
\frac{\mathrm{d} \Delta \mathrm{G}}{\mathrm{dr}}=8 \pi \mathrm{r} \gamma+4 \pi \mathrm{r}^{2} \Delta \mathrm{G}_{v}=0 \\
\mathrm{r}_{\mathrm{c}}=\frac{-2 \gamma}{\Delta \mathrm{G}_{v}}
\end{gathered}
$$

From equations $1 \& 3$ we get

$$
\Delta G_{\text {crit }}=\frac{16 \pi \gamma^{3}}{3\left(\Delta G_{v}\right)^{2}}=\frac{4 \pi \gamma r_{c}^{2}}{3}
$$




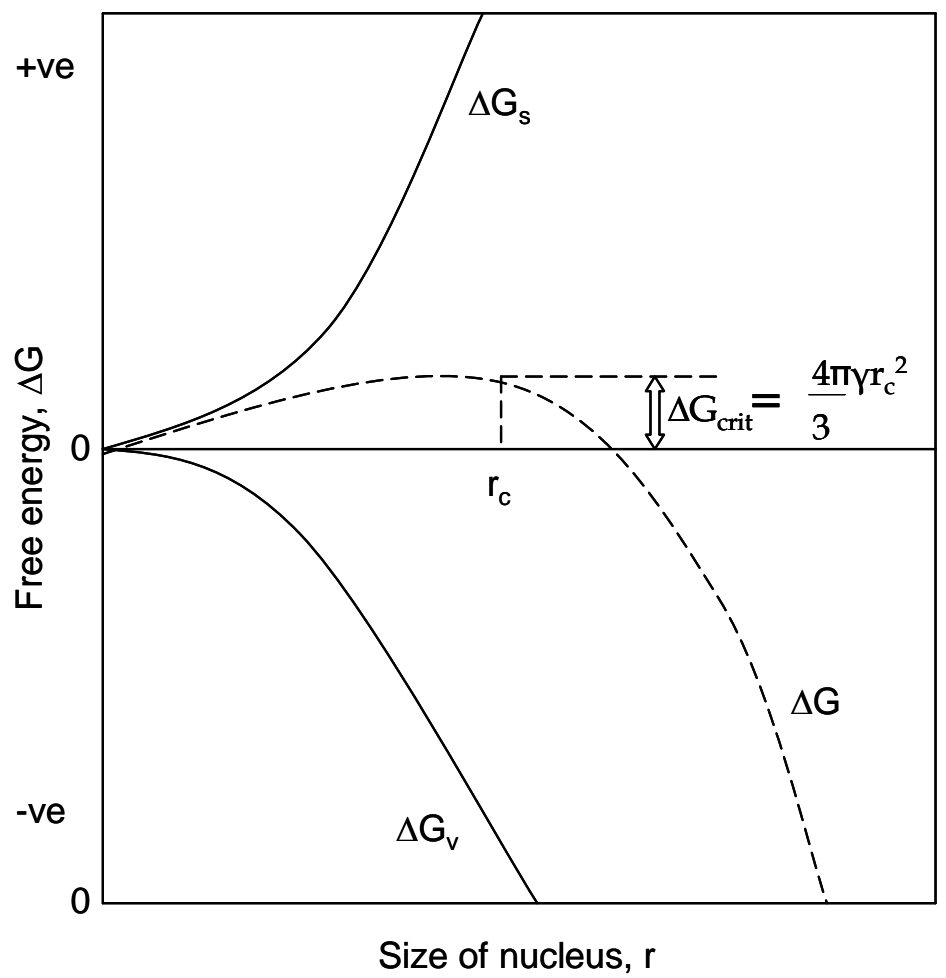

Fig. 2. Free energy diagram for nucleation explaining the existence of a 'critical nucleus'.

The behavior of a newly crystalline lattice structure in a supersaturated solution depends on its size, the crystal may grow or redissolve and it undergoes decrease in free energy of the particle. Particles smaller than $r_{c}$ will dissolve if present in a liquid in order to achieve reduction in free energy. Similar particles larger than $r_{c}$ will continue to grow.

There will be fluctuations in the energy about the constant mean value i.e. there will be a statistical distribution of energy, or molecular velocity, in the molecules constituting the system, and in those supersaturated regions where the energy level rises temporarily to a high value, nucleation will be favored.

The rate of nucleation J e.g. the number of nuclei formed per unit time per unit volume can be expressed in the form of Arrhenius reaction velocity equation

$$
J=A \exp (-\Delta G / k T)
$$

$\mathrm{k}=$ Boltzmann constant, the gas constant per molecule

The basic Gibbs-Thomson relationship for a non-electrolyte may be written as

$$
\ln S=\frac{2 \gamma v}{\mathrm{kTr}}
$$


Where $S$ is defined by equation 10

$$
S=\frac{\mathrm{C}}{\mathrm{C}^{*}}
$$

Where $\mathrm{C}$ is the solution concentration and $\mathrm{C}^{*}$ is equilibrium saturation at given temperature and $\mathrm{v}$ is the molecular volume; this gives

$$
-\Delta G_{v}=\frac{2 \gamma}{r}=\frac{k T \ln S}{v}
$$

Hence, from equation 7

$$
\Delta G_{\text {crit }}=\frac{16 \pi \gamma^{3} v^{2}}{3(k T \ln S)^{2}}
$$

And from equation 8

$$
J=A \exp \left(\frac{16 \pi v^{3} v^{2}}{3 k^{3} T^{3}(\ln S)^{2}}\right)
$$

This equation indicates that three main variables govern the rate of nucleation: temperature, $T$; degree of supersaturation, $S$; and interfacial tension, $\gamma$. Equation 13 may be rearranged to give

$$
\ln S=\left(\frac{16 \pi \mathrm{r}^{3} v^{2}}{3 \mathrm{k}^{3} \mathrm{~T}^{3} \ln (\mathrm{A} / \mathrm{J})}\right)^{1 / 2}
$$

And if, arbitrarily, the critical supersaturation, $S_{\text {crit, }}$ is chosen to correspond to a nucleation rate, $\mathrm{J}$, of say one nucleus per second per unit volume, then equation 14 becomes

$$
\ln S_{\text {crit }}=\left(\frac{16 \pi \gamma^{3} v^{2}}{k^{3} T^{3} \ln A}\right)^{1 / 2}
$$

From equation 6 and 11, the radius of a spherical critical nucleus at a given supersaturation can be expressed as 


$$
\mathrm{r}_{\mathrm{c}}=\frac{2 \gamma v}{\mathrm{kT} \ln S}
$$

For the case of non-spherical nuclei, the geometrical factor 16ח/3 in equations 7 and 12-14 must be replaced by an appropriate (e.g. 32 for cube).

Critical reviews of nucleation mechanism have been made by Nancollos and Purdie (1964), Nielsen (1964), Walton (1967), Strickland-Constable (1968), Zettlemoyer (1969), Nyvlt et al (1985) and Sohnel, Garside (1992) and Kashchiev (2000).

\subsubsection{Mechanism of growth}

Nucleation occurs over some time with constant precursor concentration. Eventually surface growth of clusters begins to occur which depletes the initial supply. When the initial concentration falls below the critical level for nucleation (critical supersaturation level), nucleation ends. A general analysis of the growth process is then important to understand nanocrystal synthesis. In general, the surface to volume ratio in smaller particles is quite high. As a result of the large surface area present, it is observed that surface excess energy becomes more important in very small particles, constituting a non-negligible percentage of the total energy. Hence, for a solution that is initially not in thermodynamic equilibrium, a mechanism that allows the formation of larger particles at the cost of smaller particles reduces the surface energy and hence plays a key role in the growth of nanocrystals. A colloidal particle grows by a sequence of monomer diffusion towards the surface followed by reaction of the monomers at the surface of the nanocrystal. Coarsening effects, controlled either by mass transport or diffusion, are often termed the Ostwald ripening process. This diffusion limited Ostwald ripening process is the most predominant growth mechanism and was first quantified by Lifshitz and Slyozov [Lifshitz and Slyozov, 1961], followed by a related work by Wagner [Wagner and Elektrochem, (1961)] known as the LSW theory.

The diffusion process is dominated by the surface energy of the nanoparticle. The interfacial energy is the energy associated with an interface due to differences between the chemical potential of atoms in an interfacial region and atoms in neighboring bulk phases. For a solid species present at a solid/liquid interface, the chemical potential of a particle increases with decreasing particle size, the equilibrium solute concentration for a small particle is much higher than for a large particle, as described by the Gibbs-Thompson equation. The resulting concentration gradients lead to transport of the solute from the small particles to the larger particles. The equilibrium concentration of the nanocrystal in the liquid phase is dependent on the local curvature of the solid phase. Differences in the local equilibrium concentrations, due to variations in curvature, set up concentration gradients and provide the driving force for the growth of larger particles at the expense of smaller particles [Sugimoto, (1987)].

\subsection{Habit modification by polymers}

\subsubsection{Habit modification by polymers of inorganic materials}

All crystal growth rates are particle size dependent and size range. For microscopic, submicroscopic particles, the size effect becomes significant.

The morphology-controlling effect of PVA is long known and utilized as a capping agent during the synthesis of nanoparticles. Using radiation chemical reduction, we have 
successfully synthesized morphology controlled copper and silver metal nanoparticles by using PVA as a capping agent. (Joshi et al.,1998, \& Temgire \& Joshi,2004) In the presence of crystal habit- modifying polymers, the crystal growth or nucleation is diverted from the non-uniform to a uniform shape. In most of the earlier studies using PVA as a crystal-habit modifier, a gel matrix made out of PVA has been used for the control of nucleation and morphology in aqueous-solution-based crystal growth. (Merrill \& Bassett, 1975, Sinha et.al., 2001, Lakshminarayanan et al., 2003, Joshi et al.,1998 \& Temigre \& Joshi,2004) Ammonium perchlorate $(\mathrm{AP})$ is one of the most extensively used solid propellant oxidizers in the propellant industry. The percentage of oxidizer in the propellant formulation varies from 70 to $80 \%$ by weight, depending on the energetic requirements and compatibility with the other ingredients. Because of the high percentage in the propellant formulation, the performance of the propellants (specific impulse and burning rate) varies with the oxidizer properties, and in turn, the performance of the oxidizer varies with the particles' size and morphology. (Sutton \& Oscar, 2001) Hence, in the present investigation, PVA has been used as a supermolecular matrix to control the morphology of AP.

$\mathrm{AP}$ is the most commonly used rocket propellant oxidizer and one of the extensively studied ammonium compounds. The morphology of the oxidizer has an important role in the formulation and performance of solid propellants, and the AP crystallized from its saturated solution gives needle-shaped crystals. The nucleation of the crystals was observed immediately after drying began. The crystals grown in the PVA showed entirely different morphologies, such as rectangular prism and rectangular wedge, in comparison to the morphologies of the AP crystals grown in the absence of PVA. The SEM images obtained are shown in Figures 3-8. Three different sets of SEM images were chosen for different concentrations, such as a low salt concentration, equal salt-polymer concentration, and high salt concentration (crystals grown from mixtures A, C, and E) (Vargeese et al.2008)

The images of the crystals grown immediately after mixing the solution and after $24 \mathrm{~h}$ of reaction time are shown in panels a and $b$ of each figure, respectively. Figures 3-5 show the images of the crystals grown from PVA 14000. Figure 3 shows that the crystals have an irregular morphology and do not have any growth orientation toward a particular plane. The images also indicate that the crystals have an irregular shape, although they tend to grow in an organized manner. This could be due to the polymer-substrate interaction that prevents the crystals from growing in an organized manner. At low salt concentrations, there is too much hydrogen bonding between the hydroxyl groups of the polymer and the hydrogen of the ammonium ion. Adsorption characteristics of polymers are different from those of other systems because of the polymers' flexibility. In addition to the usual adsorption factors, such as adsorbate-adsorbent and adsorbate-solvent interactions, a major aspect is the conformation of molecules at the interface and its role in dispersion. PVA is a flexible linear molecule with no charge and which can potentially adsorb on the surface. Bridging is considered to be a consequence of the adsorption of individual intermolecular polymer molecules on the surface. This happens through hydrogen bonding. Because of the high polymer concentration, not all the segments of the polymer are in direct contact with the surface. Also, the diffusion of ions is slow at high polymer concentrations. The possibility that the solution does not contain enough AP to grow in an organized manner cannot be ruled out. The viscous nature of the solutions containing a large quantity of PVA polymer leads to rectangular wedge- shaped crystals because diffusion is predominant and convection is suppressed for the transformation of solutes. 


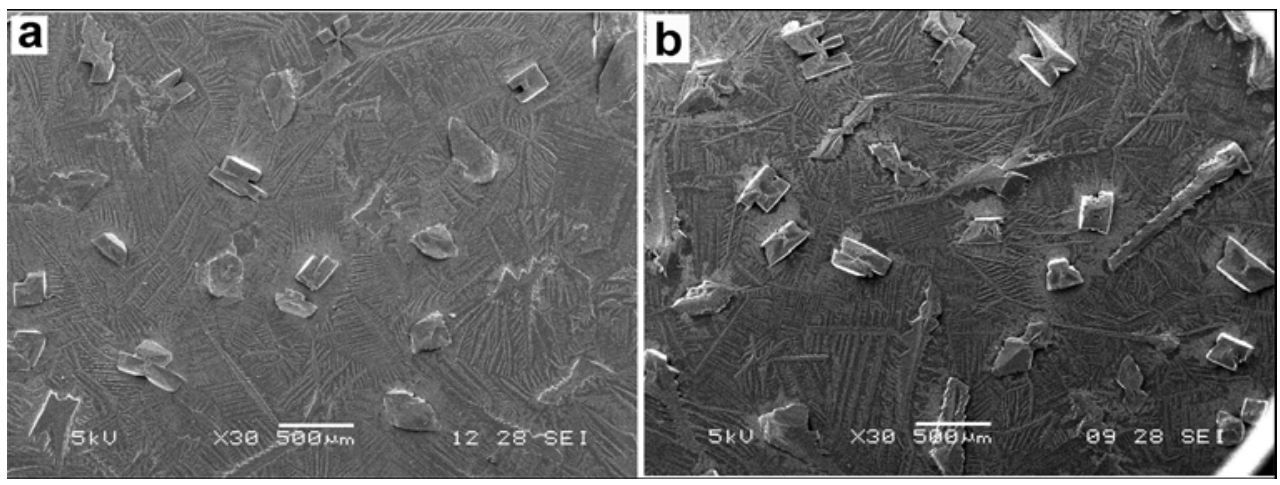

Fig. 3. SEM images of crystals grown from mixture A (PVA 14000) after (a) $0 \mathrm{~h}$ and (b) $24 \mathrm{~h}$.

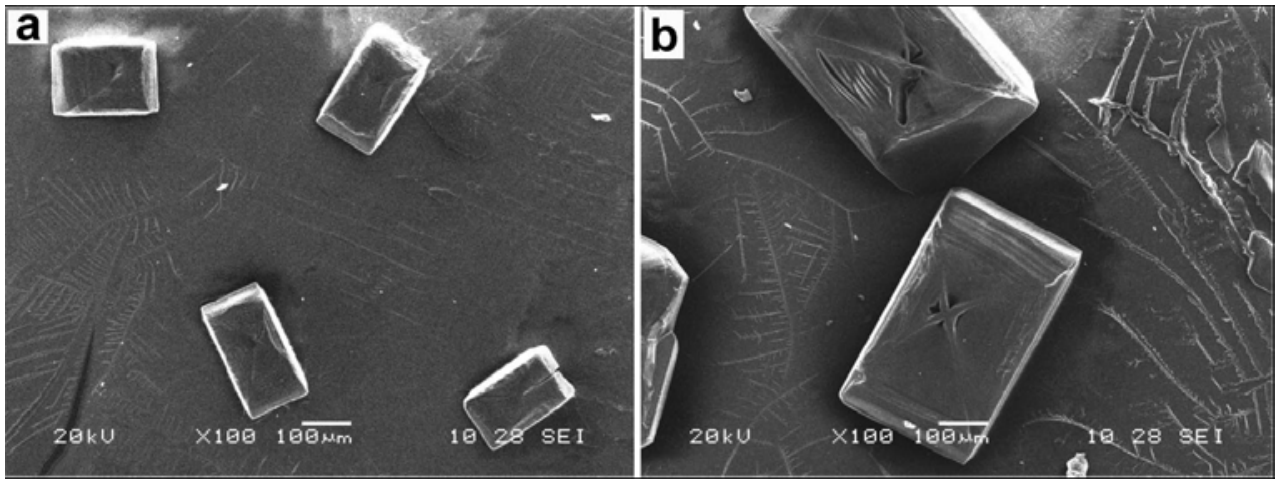

Fig. 4. SEM images of crystals grown from mixture C (PVA 14000) after (a) $0 \mathrm{~h}$ and (b) $24 \mathrm{~h}$.
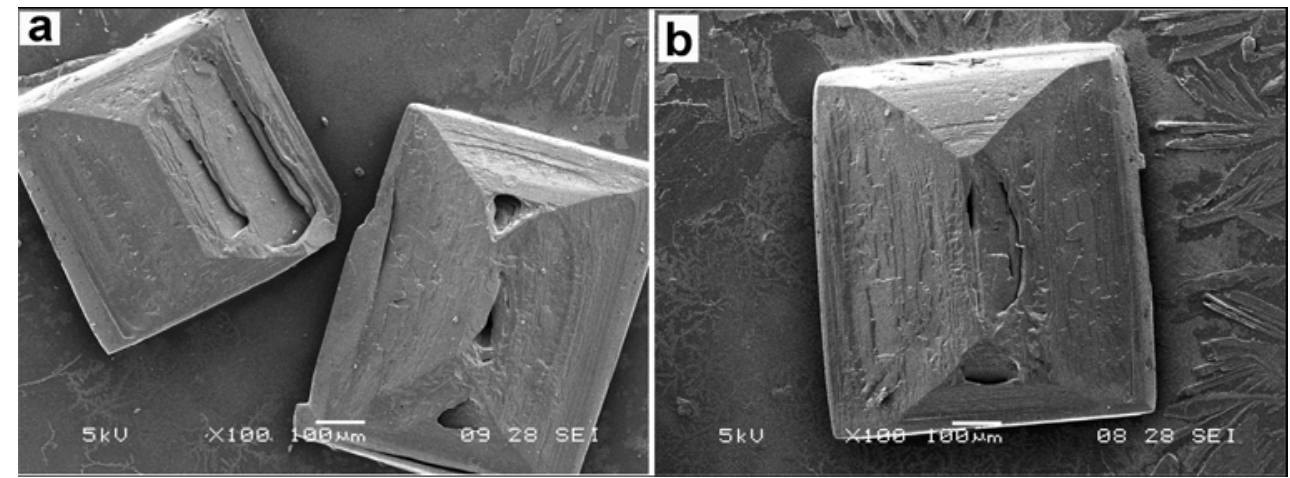

Fig. 5. SEM images of crystals grown from mixture E (PVA 14000) after (a) $0 \mathrm{~h}$ and (b) $24 \mathrm{~h}$. 


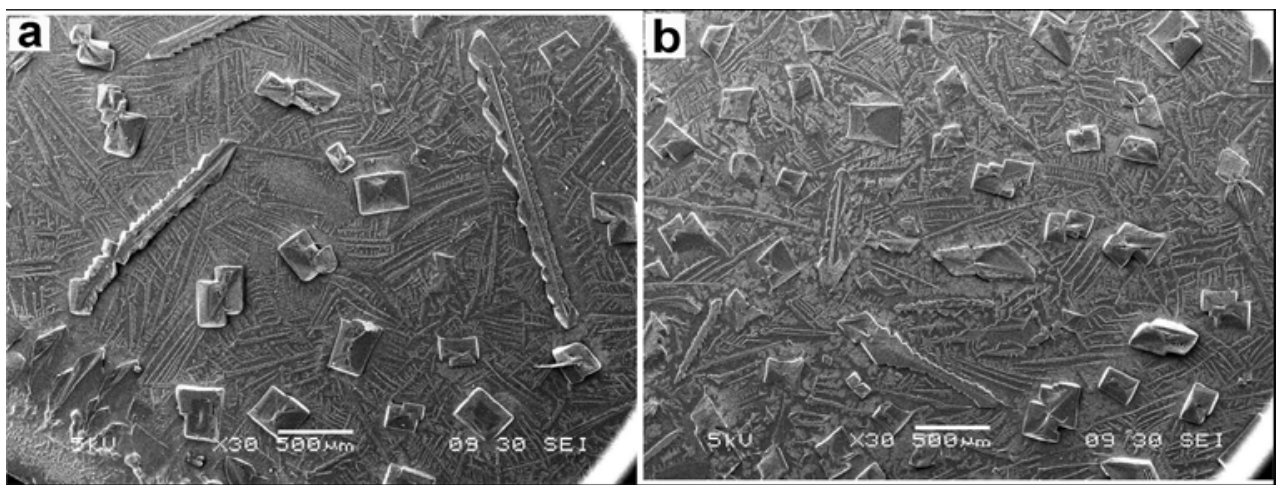

Fig. 6. SEM images of crystals grown from mixture A (PVA 125000) after (a) $0 \mathrm{~h}$ and (b) $24 \mathrm{~h}$.
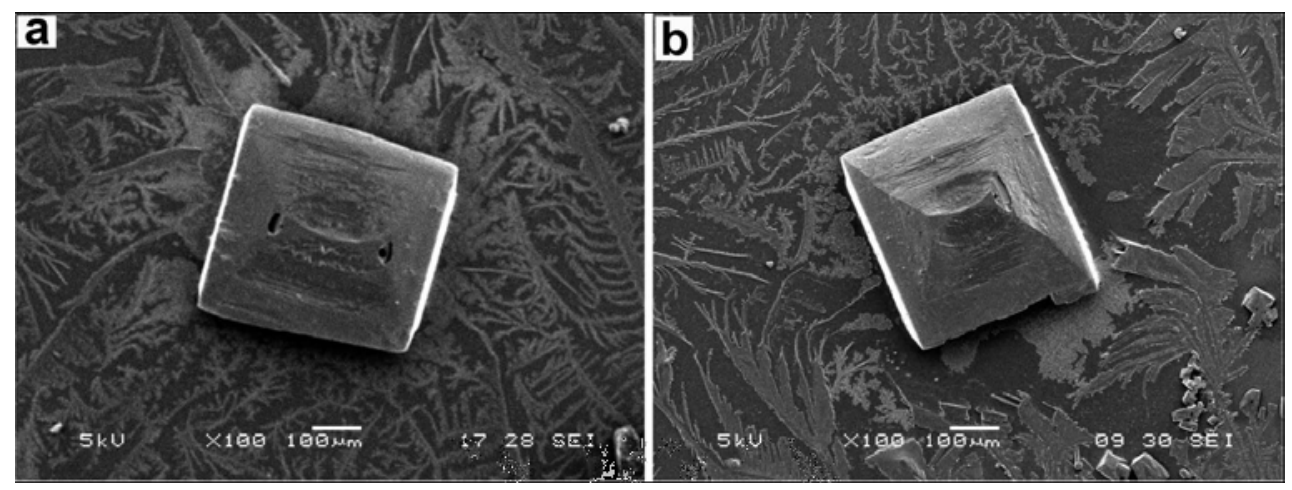

Fig. 7. SEM images of crystals grown from mixture C (PVA 125000) after (a) $0 \mathrm{~h}$ and (b) $24 \mathrm{~h}$.
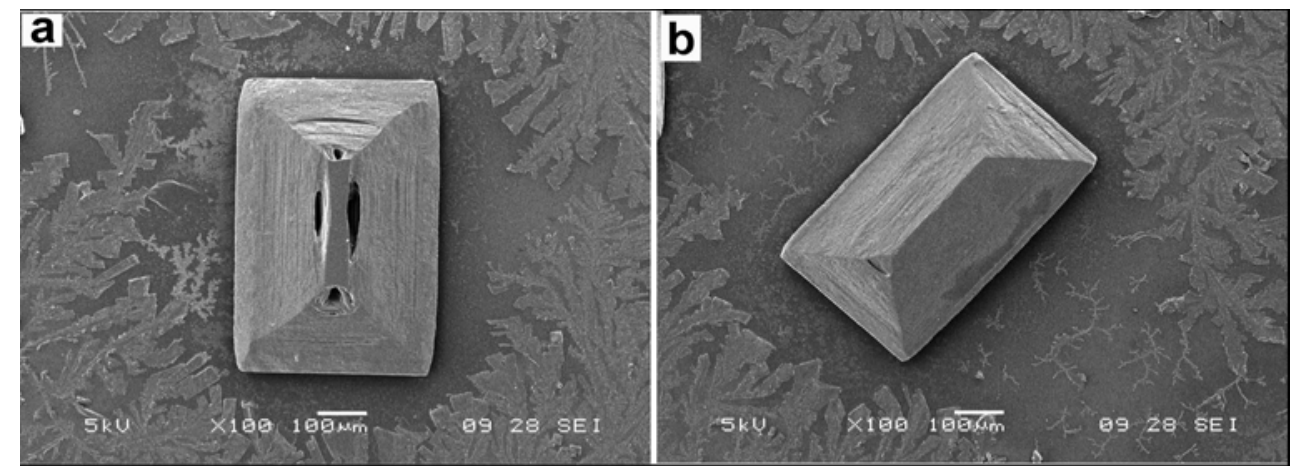

Fig. 8. SEM images of crystals grown from mixture E (PVA 125000) after (a) $0 \mathrm{~h}$ and (b) $24 \mathrm{~h}$.

As seen from the SEM images, the salt-to-polymer solution ratio change is reflected in the morphology of the crystals. Although the crystals grown from mixture A have an irregular morphology, the particles grown from the other compositions contain individual crystals with 
a specific morphology. The crystals grown from mixture $C$ have a rectangular prism shape, whereas the crystals grown from the solution containing a high salt concentration (mixture E) have a rectangular wedge shape. The crystals grown from mixtures $\mathrm{B}$ and $\mathrm{D}$ also show a more or less similar morphology, comparable to the crystals grown from mixtures $\mathrm{C}$ and $\mathrm{E}$, respectively. Hence, the crystals grown from mixtures $\mathrm{B}$ and D are not discussed here. The crystals obtained from mixture $\mathrm{E}$ have grown in another plane, leading to a rectangular wedge-like morphology in response to the change in polymer-to-salt ratio. This outgrowth to another plane of crystals obtained from mixture $\mathrm{E}$ is one of the observed modifications from the crystals grown from mixture C. The morphological evolution of the crystals from rectangular prism to wedge-like shape clearly shows the dependency of the particles' morphology on the polymer concentration and the minimum salt concentration requirement.

The advantage of using PVA as a habit modifier is a reduced agglomeration, leading to samples containing only individual particles. Studies show that, for a chemical system in which PVA and AP molecules are involved, the PVA induces the crystallization of individual AP crystals irrespectively of the polymer-substrate concentration variations. Here, the colloidal action of the PVA or the surfactant activity of the polymer could prevent the particles' agglomeration. The PVA could possibly get adsorbed on the surface, thereby preventing the agglomeration and leading to the formation of individual particles of AP. That is, immediately after the nucleation has started, the polymer might isolate the salt solution into packets and force them to grow as individual particles.

It has also been speculated that the densification of the gel medium increases the random noise for crystal growth because the polymer gel matrices disturb the progress of the growing surface. (Oaki \& Imai, 2003) The PVA gel forms an organized matrix under the optimum concentration of components, which ensures the homogeneous distribution of the cations in the polymeric network structures. The chain alignment and interchain separation of PVA, which depend on temperature and concentration, lead to the formation of a polymeric matrix with complex structures. These structures chelate the cations through a process of weak chemical bonding (such as van der Waals, hydrogen bonding) and steric entrapment. (Sinha, 2001) The polymer matrix not only provides an organized surface of mineralization, but also induces a vector growth on the polymeric surface, the direction of which differs from the characteristically preferred direction of the unit cell. (Lee et al., 1999, Addadi \&Weiner, 1992, Walsh \& Mann, 1995, Mann et al,1988) This results in the formation of extend and nonequilibrium morphologies, as well as metastable phases with lattice parameters on the order of the spacing available in the polymeric matrix. However, the nucleation of a particular space group on a charged polymeric surface not only depends on the lattice geometry, but also includes spatial charge distribution, hydration, defect sites, and surface relaxation. (Mann, 1988) These factors affect the collision frequency and in turn the activation energy for nucleation; hence, the transition state theory might be considered to explain the nucleation of biominerals. (Sinha et.al., 2001)

The shape of inorganic crystals is normally related to the intrinsic unit cell structure, and the crystal shape is usually the outside embodiment of the unit cell replication and amplification. (Yu and Colfen, 2004, Colfen \& Mann, 2003, \& Jongen et al., 2000)The diverse crystal morphologies that a mineral, identical to that for calcium carbonate, can have are due to the different surface energies and external growth environments of the crystal faces. (Wulff \&Kristallogr 1901) The morphological evolutions (from irregular to organized crystal assemblies) of the AP crystals are seen in the SEM images. The polymer-substrate 
interaction is clearly seen in the polymer pattern observed near the crystals. The polymer seems to have grown in the form of dendrites surrounding the crystals with primary and secondary branches. Usually, the rate of nucleation is governed by the temperature, the degree of supersaturation, and the interfacial tension. Crystals often grow from the center of the face and spread outward toward the edges in layers, and these layers may have a thickness of several $1000 \AA$. During this growth, dissolved impurities may affect the thickness and shape of the layers, which in turn change the morphology of the crystals. Usually, the effect of these impurities is highly specific and depends upon a number of parameters. The growth rate of a crystal face is usually related to its surface energy, if the same growth mechanism acts on each face. The fast growing faces have high surface energies, and they will vanish in the final morphology, and vice versa. This treatment assumes that the equilibrium morphology of a crystal is defined by the minimum energy resulting from the sum of the products of the surface energy and the surface area of all exposed faces (Wulff rule). (Yu \& Colfen 2004) The driving force for this spontaneous oriented attachment is that the elimination of the pairs with a high surface energy will lead to the substantial reduction of the surface free energy from a thermodynamic viewpoint. (Banfield et al., 2000 \& Alivisatos, 2000) The surface roughness on the molecular level is governed by energetic factors arising from fluid-solid interactions at the interface between the crystal and its growth environment. A change in the solvent often changes the crystal habit, and this may sometimes be explained in terms of interface structure changes. The structure of the growing crystal surface at its interface with the growth medium has an important effect on the particular mode of crystal growth adopted. A functional group with a high affinity ensures the anchoring of the molecule on a particular phase, and the polymeric chain protects the surface from coalescing with the next one through electrostatic repulsion or steric hindrance. (Joshi et al.1998) This result suggests a significant interaction between the polymeric hydroxyl groups and the crystallizing AP, resulting in the considerable influence on both the primary crystallization and the superstructure. (Qi et. al.2000)

\subsubsection{Habit modification by polymers of nanomaterials}

\subsubsection{Zinc oxide nanoparticles}

In the studies of nanomaterials, it has been observed that the size and shape of a nanomaterial depends on nature of stabilizer i.e. surfactant, ligand, polymer to salt ratio, reaction temperature and time. The synthetic method applied also plays a role. The systematic adjustment of the reaction parameters can be used to control the size and shape, a quality of nanocrystals and inorganic crystals as well. Nanoparticles are small and thermodynamically unstable. After the primary nucleation, the particles grow via molecular addition. Particles can grow by aggregation with other particles called secondary growth. Their growth rates may be arrested during the reaction either by adding surface protecting agents. Nanocrystal dispersions are stable if interaction between the capping groups and solvent is favorable providing an energetic barrier to counter act van der Waals' forces.

In our recent studies, we have synthesized flower-like $\mathrm{ZnO}$ nanostructures comprising of nanobelts of $20 \mathrm{~nm}$ width by template and surfactant free low-temperature $\left(4^{\circ} \mathrm{C}\right)$ aqueous solution route. The $\mathrm{ZnO}$ nanostructures exhibit flower-like morphology, having crystalline hexagonal wurtzite structure with (001) orientation. The flowers with size between 600 and $700 \mathrm{~nm}$ consist of $\mathrm{ZnO}$ units having crystallite size of $40 \mathrm{~nm}$. Chemical and structural characterization reveals a significant role of precursor: ligand molar ratio, $\mathrm{pH}$, and 
temperature in the formation of single-step flower-likeZnO at low temperature. Plausible growth mechanism for the formation of flower like structure has been discussed in detail. Photoluminescence studies confirm formation of $\mathrm{ZnO}$ with the defects in crystal structure. The flower-like $\mathrm{ZnO}$ nanostructures exhibit enhanced photochemical degradation of methyleneblue (MB) with the increased concentration of ligand, indicating attribution of structural features in the photocatalytic properties. (Vaishampayan et.al.2011)

$\mathrm{ZnO}$ exhibits a varied range of novel structures. The relative surface activities of various growth facets under given conditions determine the surface morphology of the grown structure. Macroscopically, a crystal has different kinetic parameters for different crystal planes, which are emphasized under controlled growth conditions. Thus, after an initial period of nucleation and incubation, a crystallite will commonly develop into a threedimensional object with well-defined, low-index crystallographic faces.

Wurtzite $\mathrm{ZnO}$ being a polar crystal, $\mathrm{Zn}$ forms a positive polar plane and $\mathrm{O}$ forms a negative polar plane. $\mathrm{Zn}^{2+}$ and $\mathrm{O}^{2}$ - ions are tetrahedrally coordinated and stack alternatively along the c-axis thus, $\mathrm{ZnO}$ grows along the c-axis. When EA is added in the aqueous solution, it gets hydrolyzed and forms EAH+ molecule. Thus, by Coulomb interaction EAH+ molecule gets adsorbed on the negative polar plane retarding the growth of $\mathrm{ZnO}$ along the negative polar plane. Therefore, when appropriate amount of EA is used, it covers the side surfaces of $\mathrm{ZnO}$ crystal, enhancing growth along the $\left(\begin{array}{lll}0 & 0 & 1\end{array}\right)$ direction. When EA concentration is lower, i.e. not enough to cover the whole surface, the Oswald ripening takes place and thereby role of $\mathrm{EAH}+$ in the growth of $\mathrm{ZnO}$ crystal results in the formation of flower-like structure where individual petal is formed by the overlay of nanobelts.

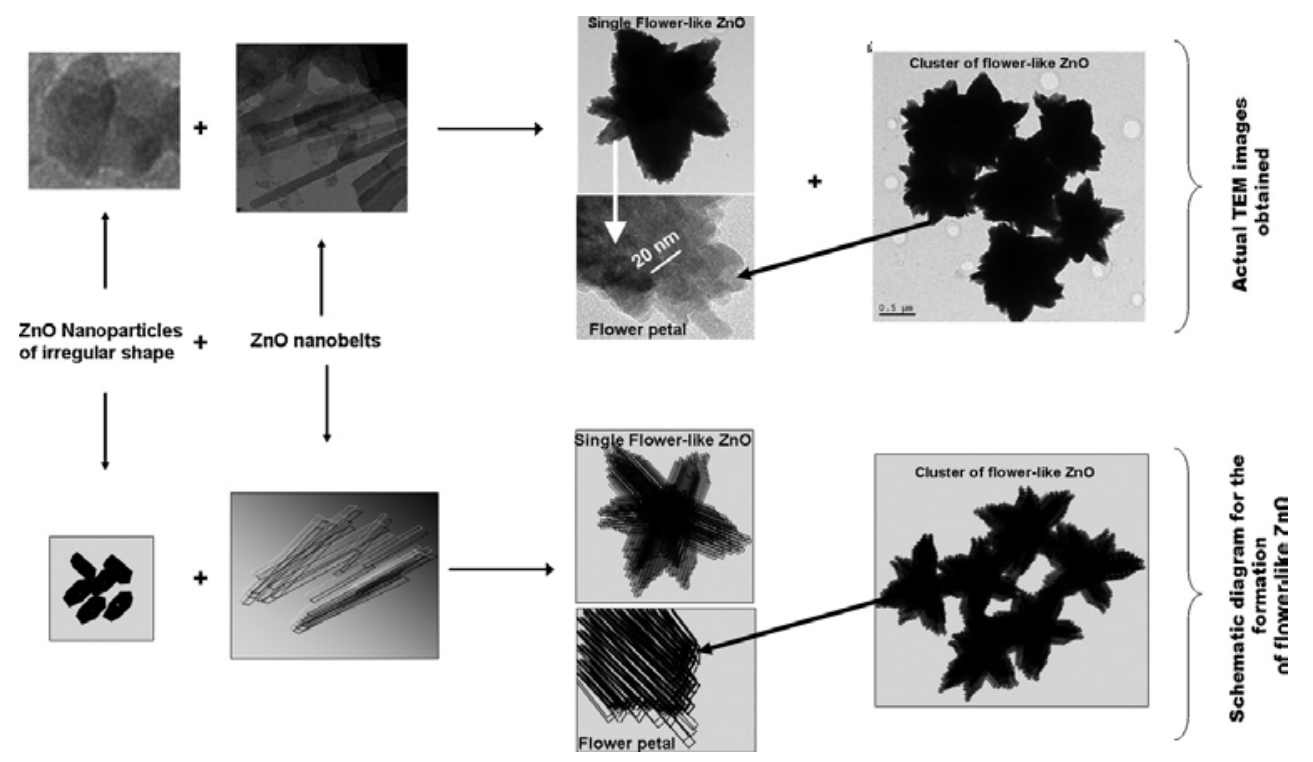

Fig. 9. Diagrammatic representation of formation of flowerlike structure.

Also, some particles of crystallite size $30 \mathrm{~nm}$ are seen on the nanobelts. The nanobelts and nanoparticles are formed by conventional nucleation followed by crystal growth process. The thermal energy released during the hydrolysis of EA facilitates nanoparticles to arrange 
themselves in between the nanobelts so as to form a compact flower-like structure. Also, as the concentration of EA increases, the nanobelts appear to have tapering feature. Thus, the ZA:EA molar ratio plays an important role in framing the morphology of the final product. The EA chelates the cations through a process of weak interactions such as van der Waals forces, hydrogen bonding, and steric entrapment. The ligand not only provides organized surface for structure formation but also induces a vector growth on the surface, the direction of which differs from characteristically preferred direction of the unit cell. This results in non-equilibrium morphologies as well as metastable phases.

We have also synthesized ZnO by aqueous thermolysis method. (Patil \& Joshi, 2007) PVA of two different molecular weights was used as a capping agent and as a fuel. A TEM study of $\mathrm{ZnO}$ nanoparticles was undertaken to highlight the shape, size and size distribution as well as the crystallinity of the particles. Fig. 10 shows the TEM image of zinc oxide nanocrystals after TGA. The micrograph of sample A (PVA 14,000) showed uniform distribution with nearly spherical morphology (Fig. 10a). All the particles are separated from each other. While sample B (PVA 125,000) synthesized with higher molecular weight exhibits cuboid like morphology and few particles appear to be close to spherical shape (Fig. 10b). In this micrograph the crystals are structurally perfect and attached like beads due to cross-linking of the polymer. This may be due to migration of defects to the surface of crystal during the calcination and growth of particles (Gu et.al, 2004) Fig. 10c shows very small particles of ZnO mostly of spherical shape for sample C (PVP 40,000). PVP does not form gel at room temperature. Therefore, it directly gets solidified while heating the precursor. As the solution is not viscous, particles formed are not cross-linked in the polymer matrix. Hence due to lack of steric interactions as compared to PVA, particles synthesized by PVP are dense but quite separated from each other. The particle sizes of XRD and TEM are comparable. The surface morphology of zinc oxide nanoparticles changes greatly with an increase in oxidation temperature. This can be clearly observed in scanning electron micrographs (SEM). Fig. 10 also shows SEM micrographs for samples A and B after TGA $\left(500{ }^{\circ} \mathrm{C}\right)$ in air. At annealing temperature of $500{ }^{\circ} \mathrm{C}$ the $\mathrm{ZnO}$ nanoparticles consist of fine grains. Since the grains are agglomerated together, grain boundaries cannot be distinguished clearly. In sample A (Fig.10d) numerous micropores were observed compared to sample B (Fig.10e). This porosity may be generated by the evolution of gases and removal of organic matter, which was loaded in the polymer network and also due to heat generated during combustion of polymers. A significant change in surface morphology is observed in the $\mathrm{ZnO}$ annealed at $1000{ }^{\circ} \mathrm{C}$ (Fig.10 f\&g), well facet grains are observed acquiring dumbbell morphology; their size becomes larger, with a wide range of distribution. At such high temperature, migration of grain boundaries occurs causing the coalescence of small grains and the formation of large grains. In order to understand the process occurring during thermolysis, we have to consider the 'cross-linking' of the polymeric network, which depends on the average molecular weight, degree of polymerization and solubility of the polymer in water. Our observation shows that PVA of molecular weight 14,000 takes almost 24 $\mathrm{h}$ to dissolve in water while higher molecular weight PVA, 125,000 dissolves in $2 \mathrm{~h}$. Both have a very good gelling property. Poly-vinyl alcohol is linear flexible molecule with no charge. Therefore, it adsorbs non-specifically on the surface of oxides. The interaction with the surface takes place through hydrogen bonds between polar functional groups of the polymer chain and hydroxylated and protonated groups on the surface. Though the interaction energy between surface and each chain segment is smaller than $k T$, chains adsorb very well because of large number of contact points. The affinity of the macromolecule for the surface usually 
increases with its molecular weight. The conformation of the adsorbed polymer remains similar to that of free macromolecule and exhibit tails and loops between contact points. The adsorbed layer provides an excellent steric protection against aggregation (Gennes, 1987, Dickson \& Ericsson, 1991). This can be schematically depicted as below.
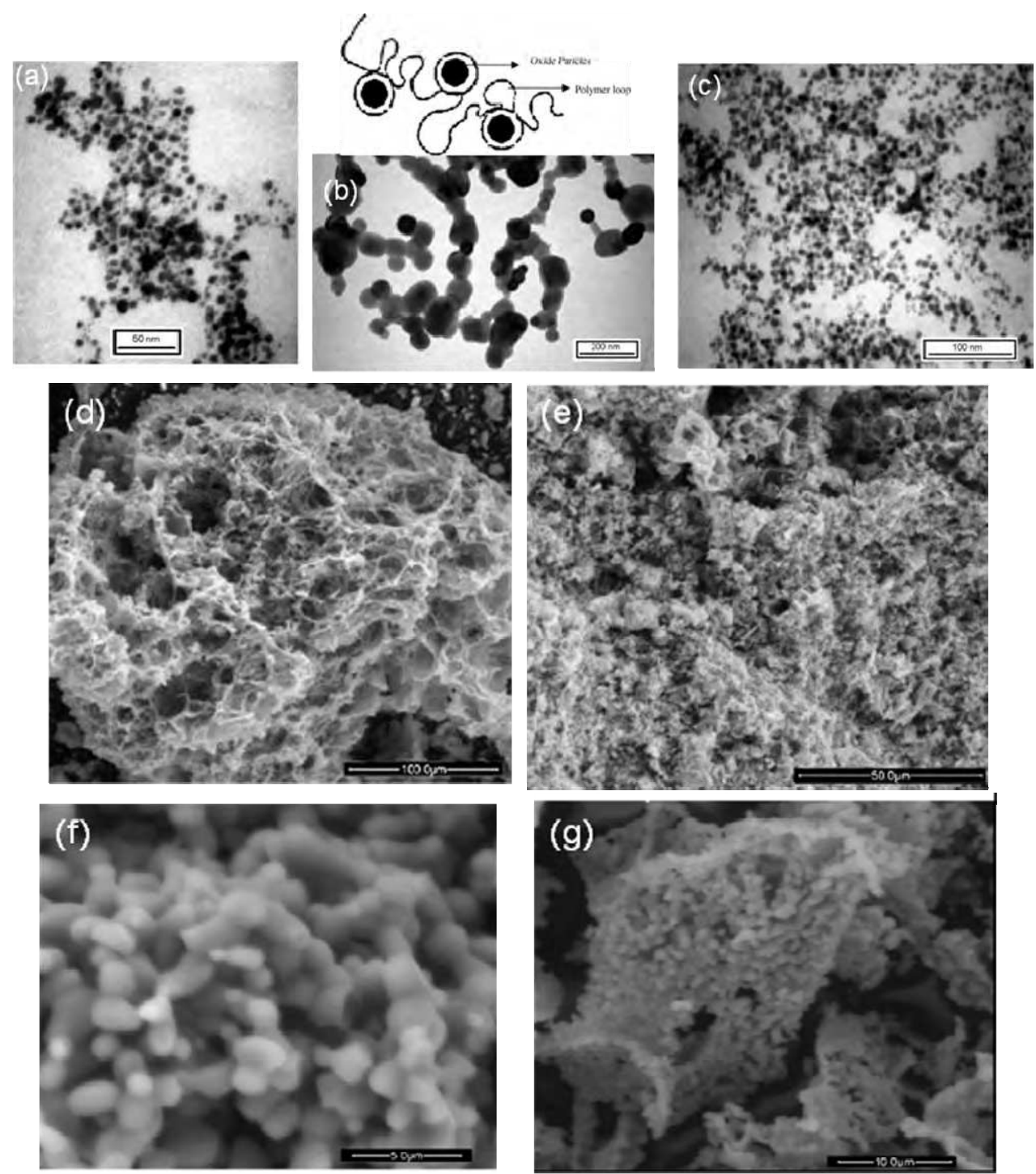

Fig. 10. TEM of ZnO for: (a) sample A, (b) sample B and (c) sample C and SEM of ZnO after TGA in air: (d) sample A at $800 \times$ magnification; (e) sample $B$ at $2000 \times$ magnification and SEM of $\mathrm{ZnO}$ after annealing at $1000{ }^{\circ} \mathrm{C}$ : (f) sample B at 12000× magnification; (g) sample $\mathrm{C}$ at $6000 \times$ magnification. 
Here polymer is adsorbed and acting as a bridge between particles. The linear chains of PVA can be cross-linked in aqueous medium, i.e. water (Kirk-Othmer, 1983). The crosslinking between the chains may provide small cages wherein the 'sol' of the reactant mixture gets trapped. During thermolysis, the 'sol' trapped in the cages may get converted to ultrafine particles of zinc oxide. Thus, the cages formed by the cross-linking may offer resistance to the agglomeration of the particles and the particle growth. The degree of polymerization can also affect the formation and morphology (Temgire \& Joshi, 2004).

\subsubsection{Palladium nanoparticles}

Stable palladium nanocluster catalysts prepared by chemical and -radiolytic reduction methods were found to give very high turn-over frequency numbers in hydrogenation of styrene oxide and 2-butyne-1,4-diol (B3D) as compared to the conventional catalysts. (Telkar et al.2004) A systematic study was carried out on the effects of different transition metals, their reduction methods, types of polymer used as a capping agent, and the concentration and composition of solvent used during catalyst preparation on the size and shape of nanoparticles. The reduction method of metal precursor directly influenced the morphology of the nanoparticles, affecting the catalyst activity considerably. The cubic-shaped nanoparticles $(5-7 \mathrm{~nm})$ were obtained in chemical reduction, while radiolytic reduction method gave spherical nanoparticles (1-5 nm).
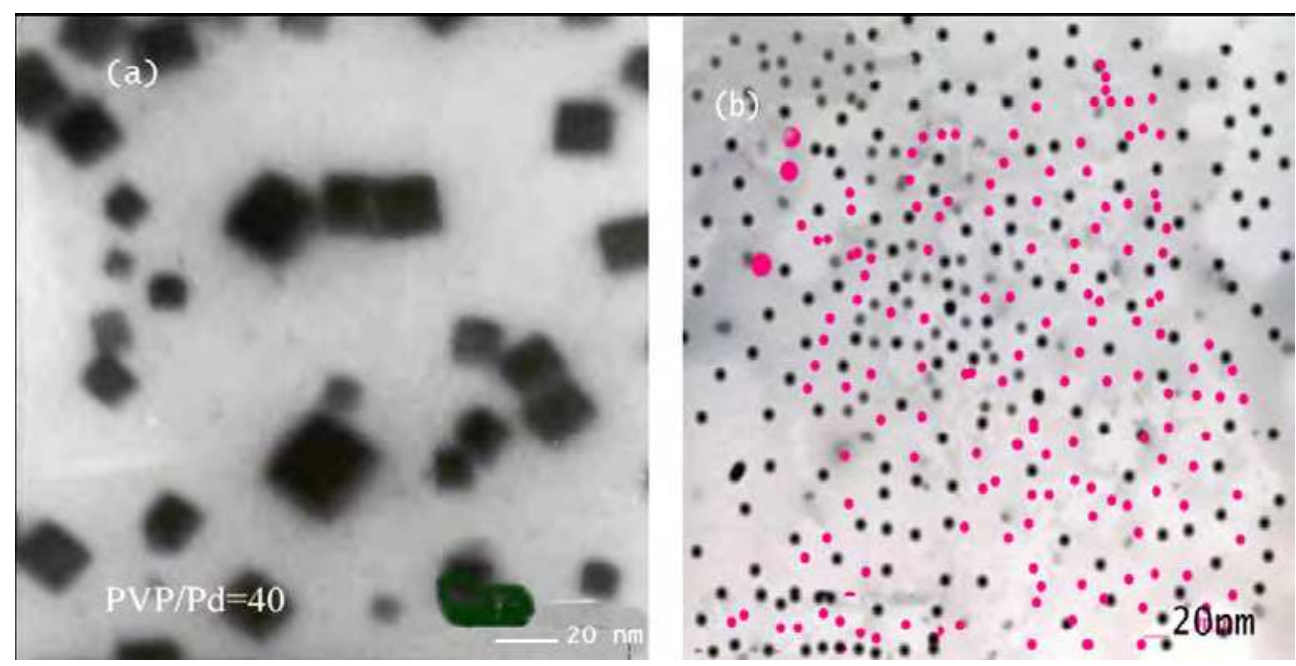

Fig. 11. TEM photograph for Pd nanoparticles prepared by (a) chemical method $(A V=80$ $\mathrm{kV}$; magnification $=40,000 \times)$ and $(\mathrm{b})$ radiolytic method $(\mathrm{PVP} / \mathrm{Pd}: 40)(\mathrm{AV}=80 \mathrm{kV}$; magnification $=80,000 \times)$. (Applied Catalysis A: General 273 (2004) 11-19)

The activity results, along with the particle sizes of various nanocluster catalysts, are presented in Table 1. The catalyst activity of RRPd (Radiolytic Reduction) catalyst was higher than that of CRPd (Chemical Reduction) catalyst when PVP/Pd ratio was 1, which was in accordance with the fact that the particle size of RRPd was less $(5.2 \mathrm{~nm})$ than that of CRPd sample $(7.1 \mathrm{~nm})$. As the PVP/Pd ratio was increased from 10 to 40, the activity trend was reversed with respect to particle sizes, thus CRPd samples showed higher catalyst 
activities than that of RRPd samples. The catalyst activity of CRPd was maximum for PVP/PD ratio of 40, in spite of the fact that particle size of RRPd sample was one-fifth of that of CRPd sample. Such a trend was consistent for both 2-butyne-1, 4-diol and styrene oxide hydrogenation. However, the extent of activity enhancement was dramatically higher for styrene oxide hydrogenation. Besides the reduction in the size, the polymer concentration seems to have a significant effect on the adsorption of reactants. It is reported that, for polymer concentration higher than $50 \mathrm{mg} / \mathrm{l}$, fully developed steric layers are formed around the particle; these act as an effective diffusion barrier that blocks further growth of the metal particles. Pd particles are considered to adsorb onto the polymer. At higher PVP/Pd ratios, beads on string-type of complexes may thus be formed, adsorbing multi-particle complexes (Boonekamp\&Kelly, 1994), which is also one of the reasons for higher activity of nanoparticles at higher PVP concentration. The selectivity to $\mathrm{B}_{2} \mathrm{D}$ obtained was more than $98 \%$ for the Pd catalysts having PVP/Pd ratio in the range of 1-30. A further increase in $\mathrm{PVP} / \mathrm{Pd}$ ratio to 40 caused a marginal decrease in 2-butene-1,4-diol selectivity from 98 to $91 \%$ due to the formation of butane-1,4-diol. In the case of catalysts prepared by the radiolytic method, the particle size reduced to $1 \mathrm{~nm}$ with increase in concentration of PVP. Surprisingly, the activity was found to reduce with decrease in particle size. This observation was consistent for both (2-butyne-1, 4-diol (B3D)) as well as styrene oxide hydrogenation (Table 1). This trend can be attributed to differences of shape of the $\mathrm{Pd}$ nanoparticles formed by chemical and radiolytic reduction methods. TEM photographs of Pd nanoparticles (Fig.11) showed a distinct morphological change depending on the method of preparation of nanoparticles. Pd particles prepared by ethanol reduction showed particles with a square outline, from which the three-dimensional shapes determined, were found to be cubic. Similar morphology was observed for $\mathrm{Pt}$ colloids prepared by $\mathrm{H}_{2}$ gas reduction. (Ahmadi et al.1996) However, they also obtained a mixture of tetrahedral, polyhedra and irregular-prismatic particles. Milligan and Morris also observed cubical gold nanoparticles for hydroxylamine hydrochloride as the reducing agent (Milligan \& Morriss, 1964). In contrast to this, the radiolytic reduction of $\mathrm{PdCl}_{2}$ gave colloidal $\mathrm{Pd}$ particles of spherical and oval shapes, 1-5 nm diameter. The final structure and size of the clusters depend on the mechanism of growth process. In the case of radiolytic reduction, the solvated electrons and $\mathrm{H}$ • are strong reducing agents and with high rate of reduction, the free metal ions are generally reduced at each encounter (Belloni et al., 1998). In the chemical reduction method, an adsorption of excess of metal ions on the reduced metal clusters, get reduced at a slower rate. This difference in reduction mechanism of radiolytic and chemical reduction may give rise to two distinct shapes of the nanoparticles. It is known that the active sites are more concentrated on the edges of the catalyst these sites may be formed in chemically reduced Pd nanoparticles, leading to higher catalyst activity for these samples. This clearly indicates that not only the particle size but also the shape of the nanoparticles influences the activity of the catalyst (Chen et al., 2000). Nanoparticles of other metals reduced by ethanol also showed cubic shapes while the radiolytic reduction gave spherical particles. As mentioned earlier, the concentration of a stabilizer influenced the nanoparticle size dramatically in case of radiolytically reduced $\mathrm{Pd}$ colloids. However, the shape remained spherical, thus confirming that the stabilizer concentration did not contribute to the shape of the nanoparticles. In order to further understand why the catalyst activity decreased in spite of considerable size reduction of radiolytically reduced Pd catalysts; stabilizing polymer alone 
was irradiated in a separate experiment. There was an increase in the viscosity of irradiated PVP, which indicates increased cross-linking of the polymer (Wang et al.1997). With increase in concentration of polymer, $(\mathrm{PVP} / \mathrm{Pd}=40)$, the polymer cross-linking may hinder the access of substrate to the Pd metal particles thereby decreasing the activity of radiolytic nanosize particles.

\begin{tabular}{|c|c|c|c|c|c|c|c|}
\hline \multirow[t]{3}{*}{$\begin{array}{l}\text { Method of } \\
\text { preparation }\end{array}$} & \multirow[t]{3}{*}{$\mathrm{PVP} / \mathrm{Pd}$} & \multirow{3}{*}{$\begin{array}{l}\text { Particle } \\
\text { size } \\
(\mathrm{nm})\end{array}$} & \multicolumn{3}{|c|}{$\mathrm{B}_{3} \mathrm{D}$ hydrogenation } & \multicolumn{2}{|c|}{$\begin{array}{l}\text { Styrene oxide } \\
\text { hydrogenation }\end{array}$} \\
\hline & & & \multirow[t]{2}{*}{$\begin{array}{l}\text { TOF } \\
\left(\times 10^{-5} h^{-1}\right)^{a}\end{array}$} & \multicolumn{2}{|c|}{$\begin{array}{l}\text { Selectivity } \\
(\%)\end{array}$} & \multirow[t]{2}{*}{$\begin{array}{l}\text { TOF } \\
\left(\times 10^{-4} h^{-1}\right) a\end{array}$} & \multirow[t]{2}{*}{$\begin{array}{l}\text { Selectivity } \\
\text { PEA (\%) }\end{array}$} \\
\hline & & & & $\mathrm{B}_{1} \mathrm{D}$ & $\mathrm{B}_{2} \mathrm{D}$ & & \\
\hline \multirow{5}{*}{$\begin{array}{l}\text { Chemical } \\
\text { method } \\
(\mathrm{CRPd})\end{array}$} & 1 & 7.1 & 3.0 & 1.0 & 99 & 1.5 & 99.9 \\
\hline & 10 & 6.1 & 3.2 & 1.8 & 98.2 & 1.4 & 99.8 \\
\hline & 20 & 6.0 & 4.3 & 1.6 & 98.4 & 3.4 & 99.6 \\
\hline & 30 & 5.5 & 4.6 & 1.6 & 98.4 & 7.0 & 99.8 \\
\hline & 40 & 5 & 5.7 & 8.8 & 91.2 & 10.2 & 99.9 \\
\hline \multirow{5}{*}{$\begin{array}{l}\text { Radiolytic } \\
\text { method } \\
\text { (RRPd) }\end{array}$} & 1 & 5.2 & 3.6 & 1.0 & 99.0 & 1.9 & 99.5 \\
\hline & 10 & 5.0 & 3.2 & 1.7 & 98.3 & 1.8 & 99.5 \\
\hline & 20 & 4.0 & 3.0 & 1.6 & 98.4 & 1.5 & 99.5 \\
\hline & 30 & 3.0 & 2.8 & 1.5 & 98.5 & 1.3 & 99.4 \\
\hline & 40 & 1 & 2.4 & 1.4 & 98.6 & 1.0 & 99.8 \\
\hline
\end{tabular}

Table 1. Effect of polymer to Pd ratio prepared by chemical and radiolytic methods for hydrogenation reactions.

\subsubsection{Copper chromite nanoparticles}

Amorphous and monodispersed copper chromite nanoparticles were prepared by aqueous thermolysis method using PVA and different ratios of urea-PVA as fuel in air (Hrishikeshi and Joshi, unpublished results, 2011). Morphology and size of nanoparticles were measured by SEM and TEM analysis. Copper chromite $\left(\mathrm{CuCr}_{2} \mathrm{O}_{4}\right)$ is a tetragonally distorted normal spinel; this distortion is due to Jahn Teller effect of $\mathrm{Cu}^{+2}\left(\mathrm{~d}^{9}\right)$ ions in tetrahedral sites. It is a p-type semiconductor which is widely used as a catalyst for the oxidation of CO (Hertl et al., 1973), hydrocarbons (Mc Cabe \& Mitchell, 1983) alcohols (Solymosi \& Krix 1962) and as a burn rate catalyst in composite solid propellants, (Prince, 1957, Solymosi \& Krix 1962, Patil et al., 2008) Well resolved square bipyramidal morphology was seen in all copper chromite samples using PVA alone. The habit modification of copper chromite was observed due to presence of urea. The urea molecule is planar in the crystal structure, but the geometry around the nitrogens is pyramidal in the gas-phase minimum-energy structure. In solid urea, the oxygen center is engaged in two N-H-O hydrogen bonds. The resulting dense and energetically favorable hydrogen-bond network probably changes the morphology after combustion process.

Figure 12 shows scanning electron micrograph of Copper chromite (a) using only PVA and (b) using Urea and PVA after annealing at $800^{\circ} \mathrm{C}$. As obtained as well as annealed samples show uniform and compact distribution of copper chromite $\mathrm{CuCr}_{2} \mathrm{O}_{4}$ nanoparticles. There is 
almost no porosity in the as obtained as well as in annealed samples. Polymer is adsorbed and acting as bridge between particles. The linear chains of PVA can be cross linked in aqueous medium (Kirk-Othmer, 1983). The cross linking between the chains may provide small cages wherein the "sol" of the reactant mixture gets trapped. During combustion, the "sol" trapped in the cages may get converted to ultrafine particles of copper chromite. Thus cages formed by the cross linking may offer resistance to the agglomeration of the particles and particle growth. Perfect square bipyramidal morphology is seen in PVA capped and orthorhombic in annealed samples. Sharpness of edges decreases gradually with increase in urea content in the fuel mixture.

\section{Square bipyramidal}

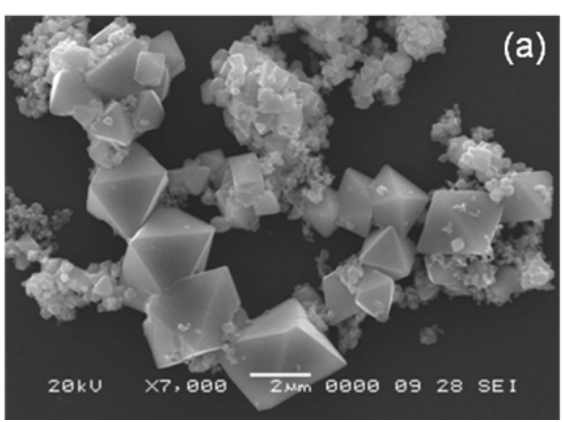

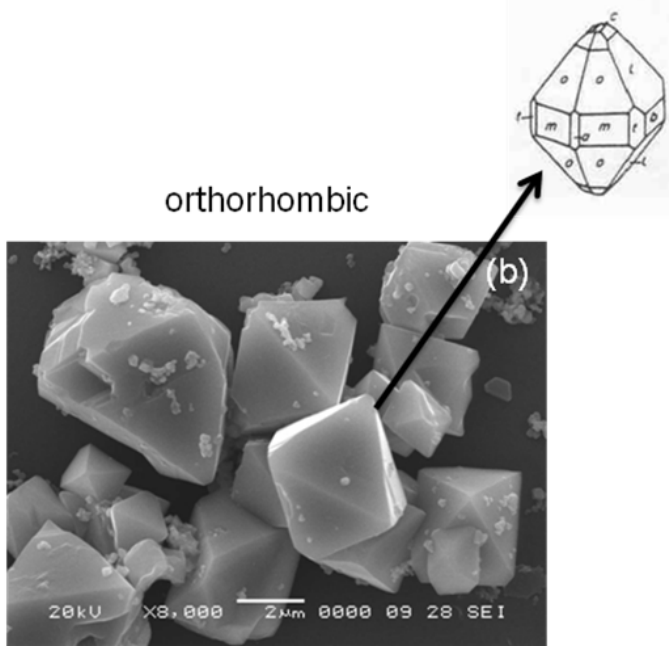

Fig. 12. SEM micrographs of Copper chromite (a) using only PVA and (b) using Urea and PVA after annealing at $800^{\circ} \mathrm{C}$.

I have tried to discuss the morphological changes and habit modification of some of the materials studied in our group, on the basis of the theories put forth and the literature.

\section{Conclusion}

In this Chapter, the habit modification and morphological changes of some inorganic materials in microsize and nanosize are discussed. In most of these studies polymers play multiple roles as a fuel in combustion synthesis, encapsulating agent and as a habit modifier in other synthesis method applied. We have observed that the size, shape, morphology of the synthesized material depends on various factors like nature of polymer, its degree of polymerization, molecular weight, reaction time, synthetic method applied and also on heat of reaction. In the methods applied at high temperature, rapid nucleation time gives rise to short burst of nuclei which might react with intermediate species and the reactions are more kinetically controlled. When the synthesis was carried out at low temperatures, nucleation process is slow and thermodynamically driven process. With aging, growth process 
becomes more favorable. Final morphology of the material depends on equilibrium conditions related to minimum surface energy, rate of nucleation and growth.

\section{Acknowledgement}

My sincere thanks are due to Ms. Tajana Jevtic, Publishing Process Manager, In Tech, for inviting me to write a chapter on the work related to crystal growth. It is rewarding to be a contributory of the book, "Crystal Growth". I take this opportunity to thank all my research students, who have worked hard and contributed to the field of Nanoscience and related area. I need to mention the effort of Ms. Shubhangi Borse, in editing the manuscript, as per the requirements of the prescribed format. I acknowledge my family members, for their cooperation, wholehearted support and constant encouragement during the preparation of this chapter.

\section{References}

Addadi, L., Weiner, S. (1992) Preparation of portland cement components by poly(vinyl alcohol) solution polymerization Angew.Chem., Int. Ed., 31, 153-169.

Ahmadi T.S., Wang Z.L., Green T.C., Henglein A., El-Sayed M.A. (1996) Shape-Controlled Synthesis of Colloidal Platinum Nanoparticles, Science 272, 1924.

Alivisatos, A. P. (2000) Naturally Aligned Nanocrystals Science, 289, 736-737.

Banfield, F., Welch, S. A., Zhang, H., Ebert, T. T. Penn, R. L. (2000) Aggregation-Based Crystal Growth and Microstructure Development in Natural Iron Oxyhydroxide Biomineralization Products Banfield, Science 2000, 289, 751-754.

Boonekamp E.P, Kelly J.J, Fokkink L.G.J (1994) Adsorption of nanometer-sized palladium particles on $\mathrm{Si}(100)$ surfaces, Langmuir, 10, 4089-4094.

Colfen, H. (2001) Double-Hydrophilic Block Copolymers: Synthesis and Application as Novel Surfactants and Crystal Growth Modifiers, Macromol. Rapid Commun., 22, 219-252.

Colfen, H., Qi, L. A. (2001) Systematic Examination of the Morphogenesis of Calcium Carbonate in the Presence of a Double-Hydrophilic Block Copolymer, Chem. Eur. J., 7, 106-116.

Colfen, H., Mann, S. (2003) Higher-Order Organization by Mesoscale Self-Assembly and Transformation of Hybrid Nanostructures, Angew. Chem., Int. Ed., 42, 23502365.

De Gennes P.G. (1987) Polymers at an interface; a simplified view, Adv. Colloid Interface Sci., 27189

Dickson E., Ericsson, L. (1991) Adv. Colloid Interface Sci., 1439.

Gibbs,J.W (1948) Collected works, Vol.1, Thermodynamics, Yale University Press,New Haven.

Gorna, K., Munoz-Espi, R., Grohn, F., Wegner, G. (2007) Bioinspired Mineralization of Inorganics from Aqueous Media Controlled by Synthetic Polymers, Macromol. Biosci., 7, 163-173.

Gu, F., Wang,S.F., Lu, M.K.,. Xu, D, Yuan. D.R. (2004) Structure evalution \& highly enhanced luminescence of $\mathrm{DY}^{+3}$-doped $\mathrm{ZnO}$ nanocrystals by $\mathrm{Li}$ doping via combustion, Langmuir 20, 3528. 
Guo, X., Yu, S., Cai, G. (2006) Crystallization in a Mixture of Solvents by Using a Crystal Modifier: Morphology Control in the Synthesis of Highly Monodisperse $\mathrm{CaCO}_{3}$ Microspheres, Angew. Chem., 118, 4081-4085.

He, L., Zhang, Y., Ren, L., Chen, Y., Wei, H., Wang, D. (2006) Double-Hydrophilic Polymer Brushes: Synthesis and Application for Crystallization Modification of Calcium Carbonate Macromol., Chem. Phys. 207, 684-693.

Henisch, H. K. (1988) In Crystals in Gels and Liesegang Rings; Cambridge University Press: Cambridge,; Chapter 2, 29-47.

Jongen, N., Bowen, P., Lemaitre, J., Valmalette, J. C.; Hofmann, H. J. (2000) Precipitation of Self-Organized Copper Oxalate Polycrystalline Particles in the Presence of Hydroxypropylmethylcellulose (HPMC): Control of Morphology, Colloid Interface Sci., 226, 189-198.

Joshi, S. S., Patil, S. F., Iyer, V., Mahamuni, S. (1998) Radiation induced synthesis and characterization of copper nanoparticles, Nanostruct. Mater., 10(7) 1135-1144.

Kashchiev, D. (2000) Nucleation, Butterworth-Heinemann Oxford.

Kirk-Othmer Encyclopedia of Chemical Technology, vol. 23, John Wiley and Sons, New York, 1983, p. 856.

Kuldipkumar, A., Tan, Y. T. F., Goldstein, M., Nagasaki, Y., Zhang, G. G. Z., Kwon, G. S. (2005) Amphiphilic Block Copolymer as a Crystal Habit ModifierKuldipkumar, Cryst. Growth Des., 5, 1781-1785.

Lakshminarayanan, R., Valiyaveettil, S., Loy, G. L., (2003) Selective Nucleation of Calcium Carbonate Polymorphs: Role of Surface Functionalization and Poly(Vinyl Alcohol) Additive, Cryst. Growth Des., 3, 953-958.

Lee, S. J., Benson, E. A., Kriven, W. M. (1999) Preparation of portland cement components by poly(vinyl alcohol) solution polymerization, J. Am. Ceram. Soc., 82, 2049-2055.

Lifshitz, I.M., Slyozov, V. V. (1961) J. Phys Chem. Solids, 19, 35

Liu, S., Yu, J., Cheng, B., Zhang, Q. (2005) Controlled Synthesis of Novel Flower-shaped $\mathrm{BaCrO}_{4}$ Crystals, Chem. Lett., 34, 564-565.

Loste, E., Meldrum, F. C. (2001) Control of calcium carbonate morphology bytransformation of an amorphous precursor in a constrained volume, Chem. Commun., 901-902.

Makoto, K. (2001) Mem. Nat. Def. Acad., Math., Phys., Chem. Eng., 1, 1-8.

Mann, S. (1988) Molecular recognition in biomineralization, Nature, 332, 119-124.

Mann, S., Heywood, R., Rajam, S., Birchall, J. D. (1988) Controlled crystallization of $\mathrm{CaCO}_{3}$ under stearic acid monolayers, Nature, 334, 692-695.

Meldrum, F. C., Ludwigs, S. (2007) Template-Directed Control of Crystal Morphologies Macromol. Biosci., 7, 152-162.

Meng, Q., Chen, D., Yue, L., Fang, J., Zhao, H., Wang, L. (2007) Hyperbranched Polyesters with Carboxylic or Sulfonic Acid Functional Groups for Crystallization Modification of Calcium Carbonate, Macromol. Chem. Phy., 208, 474-484.

Merrill, L. Bassett, W. A. (1975) The crystal structure of $\mathrm{CaCO}_{3}$ (II), a high-pressure metastable phase of calcium carbonate, Acta Crystallogr. B., 31, 343-349. 
Mullin, J. W. (2002) In Crystallization; Butterworth-Heinemann: Oxford,; Chapter 6, 216314.

Mullin. J.W., (1961) Crystallization (4th Edition Reprinted) Butterworth-Heinemann, ISBN O 75064833 3, Oxford.

Nanocallas, G.H. and Purdie, N. (1964) The kinetics of crystal growth. Quarterly Reviews of the chemical Society, 18,1-20.

Nielsen, A.E (1964) Kinetics of precipitation, Pregamon, Oxford.

Nyvlt, J. (1995) The ostwale Rule of Stages. Crystal Research and Technology, 30, 445451.

Oaki, Y., Imai, H., (2003) Experimental Demonstration for the Morphological Evolution of Crystals Grown in Gel Media, Cryst. Growth Des,. 2003, 3, 711-716.

Patil P. R., Joshi S. S., (2007) Polymerized organic-inorganic synthesis of nanocrystalline zinc oxide, Materials Chemistry and Physics,105, 354-361.

Patil P. R., Krishnamurty V. N., Joshi S. S. (2008) Effect of Nano-Copper Oxide and Copper Chromite on the Thermal Decomposition of Ammonium Perchlorate, Propellants, Explosives, Pyrotechnics, 4, 33, $266-270$.

Prince, E., (1957)Acta.Crystallogr. 10, 554

Qi, L., Colfen, H., Antonietti, M. (2000) Control of Barite Morphology by DoubleHydrophilic Block Copolymers, Chem. Mater., 12, 2392-2403.

Qi, L., Colfen, H., Antonietti, M. (2000) Crystal Design of Barium Sulfate using DoubleHydrophilic Block Copolymers, Angew. Chem., Int. Ed., 39, 604-607.

Robinson, K. L., Weaver, J. V. M., Armes, S. P., Marti, E. D., Meldrum, F. C. (2002) Synthesis of controlled-structure sulfate-based copolymers via atom transfer radical polymerisation and their use as crystal habit modifiers for $\mathrm{BaSO}_{4}, \mathrm{~J}$. Mater. Chem., $12,890-896$.

Rudolff, J., Antonietti, M., Colfen, H., Pretula, J., Kaluzynski, K., Penczek, S. (2002) DoubleHydrophilic Block Copolymers with Monophosphate Ester Moieties as Crystal Growth Modifiers of $\mathrm{CaCO}_{3}$, Macromol. Chem. Phys., 203, 627-635.

Said A.A., (1991) The role of Copper-chromium oxide catalyst in the thermal decomposition of ammonium perchlorate, J. Therm. Anal., 37,959.

Sarig, S., Mullin, J. W. (1980) Size Reduction of Crystals in Slurries by the Use of Crystal Habit Modifiers, Ind. Eng. Chem. Process Des. DeV., 19, 490-494.

Sedlak, M., Colfen, H. (2001) Synthesis of Double-Hydrophilic Block Copolymers with Hydrophobic Moieties for the Controlled Crystallization of Minerals, Macromol. Chem. Phys., 202, 587-597.

Sinha, A., Agrawal, A., Das, S. K., Ravi Kumar, B., Rao, V., Ramachandrarao, P. (2001) On the growth of monoclinic calcium carbonate in poly(vinyl alcohol), J. Mater. Sci. Lett., 20, 1569-1572.

Sinha, A., Kumar Das, S., Rao, V., Ramachandrarao, P. (2000) Synthesis of organized inorganic crystal assemblies, Curr. Sci., 79, 646-648.

Sohnel, O. and Garside, J. (1992) Precipitation: Basic Principle and Industrial Applications, Butterworth-Heinemann Oxford. 
Solymosi F., Krix E., (1962) Catalysis of solid phase reactions effect of doping of cupric oxide catalyst on the thermal decomposition and explosion of ammonium perchlorate, J.Catal., 1, 468.

Strickland-Constable, R.F (1968) Kinetics and Mechanism of crystallization, Academic Press London.

Sugimoto, T. (1987) Adv. Colloid Interface Sci., 28, 165.

Sutton, G. P., Oscar, B. (2001) In Rocket Propulsion Elements; John Wiley \& Sons: New York,; Chapter 12. 474-519.

Telkar M.M., Rode C.V., Chaudhari R.V., Joshi S.S., Nalawade A.M., (2004) Shape-controlled preparation and catalytic activity of metal nanoparticles for hydrogenation of 2butyne-1,4-diol and styrene oxide, Applied Catalysis A: General, 273, 11-19.

Temigre, M. K., Joshi, S. S. (2004) Optical and structural studies of silver nanoparticles, Radiat. Phys. Chem., 71, 1039-1044.

University Press: Cambridge, (1988) Chapter 2, 29-47.

Vaishampayan M., Joshi. S. S., Mulla I. S., (2011) Low temperature pH dependent synthesis of flower-like $\mathrm{ZnO}$ nanostructures with enhanced photocatalytic activity Material Research Bulletin, 46(5), 771-778.

Vargeese. A. A., Joshi. S. S., Krishnamurthy V. N. (2008) Role of Poly(vinyl alcohol) in the Crystal Growth of Ammonium Perchlorate, Crystal Growth E Design, 8, 3, 1060-66.

Volmer,M. (1939) Kinetic der Phasenbildung, Steinkopff, Leipzig.

Wagner, C. (1961) Elektrochem.Z, 65, 581

Walsh, D., Mann, S. (1995) Molecular recognition in biomineralization, Nature, 377, 320 323.

Walton.A.G. (1967) The formation and properties of precipitates, Interscience, New York.

Wang, F., Xu, G., Zhang, Z., Xin, X. (2005) Morphology control of barium sulfate by PEOPPO-PEO as crystal growth modifier, Colloids Surf., A, 259,151-154.

Wang, T., Rother, G., Colfen, H. (2005) A New Method to Purify Highly Phosphonated Block Copolymers and Their Effect on Calcium Carbonate Mineralization, Macromol. Chem. Phys., 206, 1619-1629.

Wulff, G. Z. (1901) On the question of the rate of growth and dissolution of crystal surfaces, Kristallogr. Mineral., 34, 449-530.

Xu, A., Ma, Y., Colfen, H. (2007) Biomimetic mineralization, J. Mater. Chem., 17, 415-449

Yu, J. G., Zhao, X. F., Liu, S. W., Li, M.; Mann, S., Ng, D. H. L. (2007) Poly(methacrylic acid)mediated morphosynthesis of $\mathrm{PbWO}_{4}$ micro-crystals, Appl. Phys. A: Mater. Sci. Process., 87, 113-120.

Yu, J., Liu, S., Cheng, B. (2005) Effects of PSMA additive on morphology of barite particles, J. Cryst. Growth., 275, 572-579.

Yu, S., Colfen, H. (2004) Bio-inspired crystal morphogenesis by hydrophilic polymers, J. Mater. Chem., 14, 2124-2147.

Yu, S., Colfen, H., Antonietti, M. (2002) Control of the Morphogenesis of Barium Chromate by Using Double-Hydrophilic Block Copolymers (DHBCs) as Crystal Growth Modifiers, Chem. Eur. J., 8, 2937-2945. 
Yu, S., Colfen, H., Antonietti, M. (2003) Polymer-Controlled Morphosynthesis and Mineralization of Metal Carbonate Superstructures, J. Phys. Chem. B, 107, 73967405.

Zettlemoyer, A.C (ed.) (1969) Nucleation, Dickker, New York. 


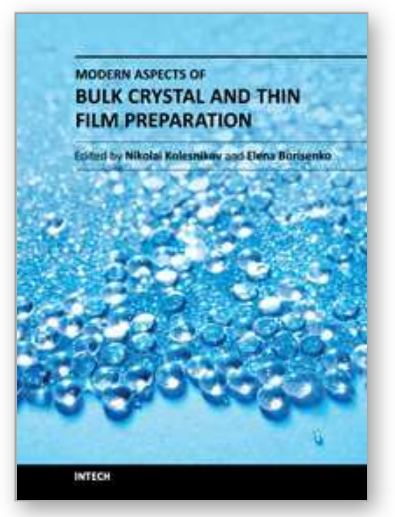

\author{
Modern Aspects of Bulk Crystal and Thin Film Preparation \\ Edited by Dr. Nikolai Kolesnikov
}

ISBN 978-953-307-610-2

Hard cover, 608 pages

Publisher InTech

Published online 13, January, 2012

Published in print edition January, 2012

In modern research and development, materials manufacturing crystal growth is known as a way to solve a wide range of technological tasks in the fabrication of materials with preset properties. This book allows a reader to gain insight into selected aspects of the field, including growth of bulk inorganic crystals, preparation of thin films, low-dimensional structures, crystallization of proteins, and other organic compounds.

\title{
How to reference
}

In order to correctly reference this scholarly work, feel free to copy and paste the following:

Satyawati S. Joshi (2012). Crystal Habit Modification Using Habit Modifiers, Modern Aspects of Bulk Crystal and Thin Film Preparation, Dr. Nikolai Kolesnikov (Ed.), ISBN: 978-953-307-610-2, InTech, Available from: http://www.intechopen.com/books/modern-aspects-of-bulk-crystal-and-thin-film-preparation/crystal-habitmodification-using-habit-modifiers

\section{INTECH}

open science | open minds

\author{
InTech Europe \\ University Campus STeP Ri \\ Slavka Krautzeka 83/A \\ 51000 Rijeka, Croatia \\ Phone: +385 (51) 770447 \\ Fax: +385 (51) 686166 \\ www.intechopen.com
}

\author{
InTech China \\ Unit 405, Office Block, Hotel Equatorial Shanghai \\ No.65, Yan An Road (West), Shanghai, 200040, China \\ 中国上海市延安西路65号上海国际贵都大饭店办公楼405单元 \\ Phone: +86-21-62489820 \\ Fax: +86-21-62489821
}


(C) 2012 The Author(s). Licensee IntechOpen. This is an open access article distributed under the terms of the Creative Commons Attribution 3.0 License, which permits unrestricted use, distribution, and reproduction in any medium, provided the original work is properly cited. 